Check for updates

Cite this: Phys. Chem. Chem. Phys., 2018, 20, 7559

Received 12th December 2017 Accepted 6th February 2018

DOI: $10.1039 / c 7 c p 08334 c$

rsc.li/pccp

\section{Associative behaviour and effect of functional groups on the fluorescence of graphene oxide $\dagger$}

\author{
Sefika Ozcan, (D) ab Sesha Vempati, (D)*a Ali Çırpan (D) *bcde and Tamer Uyar (D)*af
}

We have juxtaposed the structural, vibrational and emission properties of graphene oxide (GO) with various degrees of reduction with and without a model dispersant, unveiling a strong associative behavior between GO sheets and the influence of $\mathrm{H}$-bonds. The interlayer spacings are $\sim 0.84$ and $0.78 \mathrm{~nm}$ for the as prepared and reduced samples. $-\mathrm{OH}$ groups are predominantly effected by the photo-thermal reduction. Also we note some regeneration of $=\mathrm{C}=\mathrm{O}$ and $-\mathrm{COOH}$ groups in reduced samples. Clear changes to the phonon density of states indicated the doping effects due to $\mathrm{H}$-bonds via the oxygeneous groups. Importantly, the defect related Raman bands are rather prone to the effect of dispersant, unveiling their intrinsic nature. In the context of fluorescence, internal vibration relaxation mediated by $\mathrm{CC}$ stretch vibrations emphasized the localized nature of $\mathrm{sp}^{2}$ domains of relatively smaller size. Fluorescence consists of 6 components, where the higher energy components are more influenced due to $\mathrm{H}$-bonds than those of the lower energy regime, attributed to their associative behavior and chemical functionality, respectively. Excitation dependent fluorescence measurements indicated a range of optical gaps from $\sim 3.5$ to $2 \mathrm{eV}$. The associative behavior of $\mathrm{GO}$ and $\mathrm{rGO}$ with and without a dispersant provides crucial insights into the fundamental understanding of various molecular processes.

\section{Introduction}

Oxidation of graphite $e^{1,2}$ and reduction of the oxidized form ${ }^{3-6}$ have been extensively studied due to their vast spectrum of applications such as optoelectronics, ${ }^{7,8}$ fluorescence microscopy, ${ }^{9-11}$ etc. This reduction followed by oxidation enhances the applicability due to the scalability of the process. Researchers have devised reduction methods based on thermal, ${ }^{4,5}$ photothermal, ${ }^{6}$ and chemical $^{5}$ processes. It is known that when graphene oxide (GO) is reduced the total density of various oxygeneous functional groups decreases. However, the fractional contribution of each type of group is dependent on the reduction process, where the reactivity is determined by the chemical

${ }^{a}$ UNAM-National Nanotechnology Research Center, Bilkent University,

Ankara06800, Turkey.E-mail:svempatio1@qub.ac.uk, uyar@unam.bilkent.edu.tr

${ }^{b}$ Department of Polymer Science and Technology, Middle East Technical University, Ankara 06800, Turkey

${ }^{c}$ Department of Chemistry, Middle East Technical University, Ankara 06800, Turkey.E-mail: acirpan@metu.edu.tr

${ }^{d}$ Department of Micro and Nanotechnology, Middle East Technical University, Ankara 06800, Turkey

${ }^{e}$ The Centre for Solar Energy Research and Application (GÜNAM), Middle East Technical University, 06800 Ankara, Turkey

${ }^{f}$ Institute of Materials Science \& Nanotechnology, Bilkent University, Ankara 06800, Turkey

$\dagger$ Electronic supplementary information (ESI) available: SEM images, UV-reduction mechanism, XPS survey analysis, and FTIR and fluorescence spectra. See DOI: $10.1039 / \mathrm{c} 7 \mathrm{cp} 08334 \mathrm{c}$ environment and the functional group itself. ${ }^{12}$ In the context of GO/partially reduced GO (rGO), the (residual) oxygeneous groups and defects are the active sites, which make the surface rather functional. This functionality leads to tunable properties via associative/self-assembly-like behavior ${ }^{7,8}$ such as the formation of graphene intercalating compounds with a range of materials like poly(aniline), ${ }^{7}$ water,${ }^{13}$ methanol, ${ }^{13}$ phosphonium ions, ${ }^{14}$ etc. The associative behavior of rGO depicted better fluorescence quenching efficiency than that of GO, ${ }^{9}$ where an improved $\pi-\pi$ stacking between the fluorescent species and GO increases its efficiency. ${ }^{15}$ The quenching is, in fact mediated by Förster resonance energy transfer (FRET), or non-radiative dipole-dipole coupling. ${ }^{9-11}$ However, notably, solvent influences the associative behavior and of course the optoelectronic properties. For instance, FRET is dependent on the distance between the interacting species. Essentially, there is an explicit competition between (i) cohesive interactions of $\mathrm{sp}^{2}$ domains within $\mathrm{GO} / \mathrm{rGO}$ and (ii) those of $\mathrm{sp}^{2}$ domains of $\mathrm{GO} / \mathrm{rGO}$ and any other fluorescent species. Furthermore, point (i) can be divided into 2 cases based on the (a) presence and (b) absence of a dispersant, where the consequences of the self-association of $\mathrm{sp}^{2}$ domains of GO/rGO can be unveiled. On the other hand, the self-association is a vital phenomenon, that is governed by the dispersant (hydrogen bonds), and the density and distribution of functional groups on GO/rGO. Notably, the dispersant with varying $\mathrm{pH}$ has severe impact on the fluorescence properties of GO. ${ }^{16}$ In this case, the emission is interpreted as occurring from 
quasi-molecular species while the fluorescence is influenced by $\mathrm{pH}$ via $\mathrm{COOH}$ groups. ${ }^{16}$

In line with the above arguments to unfold the complex phenomenon of the self-association of $\mathrm{sp}^{2}$ domains and its influence on the structural, vibrational and fluorescence properties, we interrogate GO, and rGO for the above (a) and/or (b) cases. We choose $\mathrm{H}_{2} \mathrm{O}$ as a model dispersant while the density and distribution of functional groups are highly dependent on the reduction-process ${ }^{17}$ and their parameters. ${ }^{6}$ To prepare rGO we have chosen the photo-thermal ${ }^{6}$ reduction process due to its controllability and chemical purity of the products. ${ }^{17}$ In this work, after a careful juxtaposition and deeper analyses of cases (a) and (b) for GO partially reduced to various degrees, we unveiled vital information that will enhance the overall understanding of the system, enabling further development.

\section{Experimental}

\section{Materials and methods}

Preparation of GO. GO was synthesized using a method described in the literature, wherein it is referred to as an 'improved' method by Marcano et $a .^{2}$ All chemicals were of analytical grade and obtained from Sigma Aldrich. GO was extracted and dispersed in water $\left(0.5 \mathrm{mg} \mathrm{mL}^{-1}\right)$ to yield a clear brown solution.

Preparation of rGO. $0.5 \mathrm{mg} \mathrm{mL} \mathrm{m}^{-1}$ dispersions were heated to and maintained at $70{ }^{\circ} \mathrm{C}$ in a closed container where UV-Vis light (300 W, Ultra-Vitalux lamp) was illuminated for 3, 6 and 9 hours. These samples were referred to as rGO3, rGO6 and rGO9, respectively. The distance between the lamp and GOdispersions was $\sim 12 \mathrm{~cm}$. The reduced and GO dispersions were used as prepared for spectroscopic/other studies. On the other hand, the dispersions were drop-cast and dried multiple times to form films on quartz substrates. The thicknesses of the films were estimated to be $\sim 0.01 \mathrm{~mm}$. We used these films for further characterization. Fluorescence yields and spectral features may be dependent on the quantum confinement effects which we expect for a limited number of layers. Since our films are as thick as $0.01 \mathrm{~mm}$, this dependency diminishes and our fluorescence results represent bulk characteristics. TEM images for the GO and rGO samples were obtained from an FEI-Tecnai G2F30 transmission electron microscope by drying a drop of dispersion on copper grids. To investigate the changes to the crystalline structure of graphite due to oxidation (GO) and subsequent reduction (rGOx) we recorded XRD patterns within the $2 \theta=0^{\circ}-55^{\circ}$ range (PANalytical X'Pert Multipurpose X-ray Diffractometer, $\mathrm{CuK} \alpha=1.5418 \AA$ A). UV-Vis absorption measurements for the GO and rGO dispersions (without dilution) were performed with a Cary 100 UV-Vis spectrophotometer. The surface chemical properties of the samples were investigated by X-ray photoelectron spectroscopy (XPS, Thermo Scientific $\mathrm{K}-\mathrm{Alph}$, $\mathrm{Al} \mathrm{K} \alpha$ radiation with $h \nu=1486.6 \mathrm{eV}$ ) in the presence of a flood gun charge neutralizer. The X-ray spot size was $400 \mu \mathrm{m}$ in diameter. For the core-level spectra, the pass energy and step size were set to $30 \mathrm{eV}$ and $0.1 \mathrm{eV}$, respectively.
Spectral deconvolutions of the XPS data were performed through Avantage software (with a Shirley background). This deconvolution disentangles the fractional contribution of each of the ionic states to the total density of states (DOS). In the present context, variations in the DOS from C1s core-level spectra were significant. To determine the fractional contribution of each of the functional group peak deconvolution is generally employed. However, the peak fit consists of a number of free parameters such as peak position, full width at half maximum (fwhm), Lorentzian to Gaussian ratios (L/G) for each peak. Due to the number of free parameters the fitting procedure would be less reliable to determine the correct fractional contribution of functional groups in the reduced samples. To overcome this scenario, the peak parameters (fwhm and $\mathrm{L} / \mathrm{G}$ ) for each of the fractional DOS for the GO sample were set on the reduced counterparts and not allowed to vary. Nevertheless, the spectral shift is rather useful to understand the chemistry of the surface. We acknowledge that our constraint assumes invariant spectral width; however, the consistent results supported that the assumption is in fact valid. FTIR spectra were obtained from a Bruker VERTEX70 FTIR Spectrometer. The measurements were performed by pelletizing the GO or rGOx films in IR grade $\mathrm{KBr}$. The spectra were recorded between 4000 and $400 \mathrm{~cm}^{-1}$ with a resolution of $4 \mathrm{~cm}^{-1}$ for a total 128 scans. Background subtracted spectra were used for the deconvolution of the $-\mathrm{OH}$ band and also to calculate the peak height ratios. Fluorescence (Jobin Yvon, FL-1057 TCSPC) measurements were performed on films (dried on quartz pieces) and dispersions (without any dilution) with different excitation wavelengths. Raman spectroscopy was performed on both dispersed and film samples with WITec instruments (Alpha 300S, $532 \mathrm{~nm}$ laser). Origin 8.5 was employed for the analyses of Raman and FTIR spectra. The difference in Raman response (rGOx - GO) was obtained after subtracting a linear background followed by a normalization.

\section{Results and discussion}

Graphene oxide depicts interesting optoelectronic properties; however, many of them can be better understood through a controlled reduction. On the other hand, we can harness the tunability of the opto-electronic properties via reduction, which, however, depend on the chemical structure of GO. ${ }^{13} \mathrm{C}-\mathrm{NMR}$ results showed that the carbon basal plane contains epoxy and hydroxyl groups, while the edges contain carboxy, ketone, lactol, and phenol groups. ${ }^{18}$ Due to spatial restrictions and the requirement of $\mathrm{C}-\mathrm{C}$ bond cleavage, carboxyl and ketones are expected at the edges. Importantly, this functionalization opens the band gap of graphene. ${ }^{19}$ The photo-thermal reduction subjects the oxygeneous functional groups to chemical reactions and subsequent reduction of GO. UV illumination excites graphene oxide $\left(\mathrm{GO}^{*}\right)$, creating electron/hole pairs, and leads to the formation of radicals $\left(\mathrm{GO}^{\bullet}\right)$. Meanwhile, the hole reacts with water, resulting in the dissociation of protonic hydrogen and the hydroxyl ion. The ionized water $\left(\mathrm{H}^{+}\right.$and $\left.\mathrm{OH}^{-}\right)$further reacts 
with the oxygeneous functional groups of GO. When GO is reduced with $\mathrm{UV}, \mathrm{sp}^{3}$ bonded oxygen bearing functional groups such as $-\mathrm{COH}$ and $\mathrm{C}-\mathrm{O}$ can be more easily cleaved than $\mathrm{C}=\mathrm{C}$ and $\mathrm{O}-\mathrm{C}=\mathrm{O}$ due to their higher bond dissociation energies. ${ }^{20}$ This reaction leads to the removal of oxygeneous functional groups and thus the reduced form of GO. ${ }^{21}$ Please refer to Box S1 (ESI $\dagger$ ) and references therein for some proposed photoassisted reduction mechanisms. We also note that the starting material (relative level of oxidation) has a crucial role to play in determining the functional distribution, density and optoelectronic properties.

HRTEM images for GO and rGO3 are shown in Fig. 1a and b, respectively. We have observed significant differences in the contrasts of the images, which we attribute to the variations in the number of layers that were stacked and/or folded. It is notable that the $\mathrm{sp}^{2}$ sites come together and form an ordered aggregation as observed by Rani et al. ${ }^{22}$ However, the lighter regions reveal a successful chemical exfoliation of graphene sheets. Apart from this, a sheet like morphology is observed for all samples. This observation is consistent with the literature, where severe oxidation can exfoliate the graphite oxide into graphene oxide in the dispersion state.,23,24 For instance, refer to the discussion related to Fig. 3 and 5 of ref. 2 and 23 respectively.

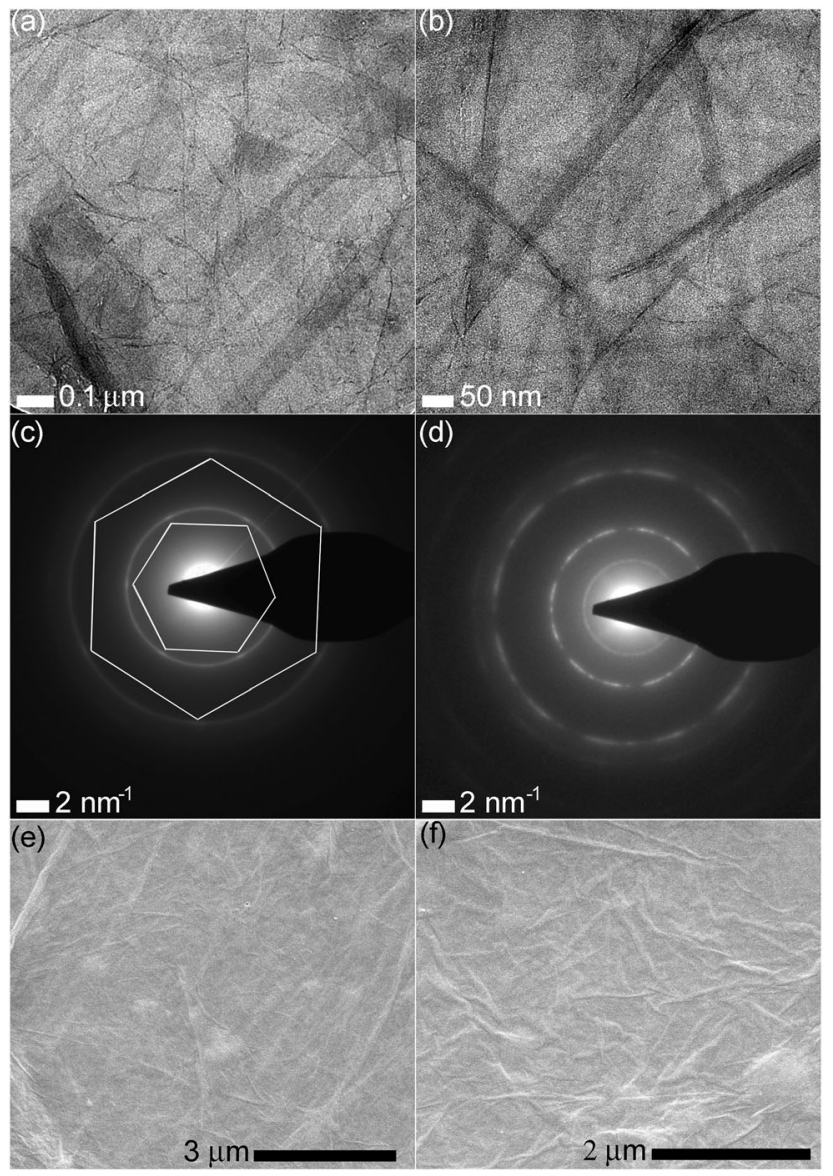

Fig. 1 TEM images of $\mathrm{GO}$ and after reduction for 3 hours (rGO3), 6 hours (rGO6) and 9 hours (rGO9): (a) GO, (b) rGO3, (c) rGO6 and (d) rGO9. SEM images of (e) rGO6 and (f) rGO9 samples.
However, despite TEM being a local probe, we will see that the exfoliation is indeed successful and the associative behavior will be explicit in the context of fluorescence. Furthermore, to understand the local crystalline properties, SAED patterns of the rGO6 and rGO9 samples are shown in Fig. 1c and d, respectively. The SAED patterns of rGO6 and rGO9 indicate the graphite or its nonexfoliated oxide. ${ }^{23}$ We will see a signature of a small fraction of $\mathrm{AB}$ stacking in the context of XRD, which is a bulk-averaged measurement determined by the volume of the sample and probe depth of the X-rays. For rGO6 we noted 6 spots (i.e. bright spots in a triangular geometry), which indicates the hexagonal lattice of graphite and corresponds to the (110) diffraction plane. The diffraction spots corresponding to (020) make $30^{\circ}$ with respect to that of (110). On the other hand, the variations in the nuclear geometry reflect the size of the spots in the diffraction pattern, which seemed to be sharper for rGO9 than that of rGO6. If the SAED patterns are actually from graphite oxide, then the decreased density of oxygeneous functional groups lowers the strain in $\mathrm{C}-\mathrm{C}$ bonds. Hence, it may be convincing that the fluctuations in the nuclear geometry are lower for rGO9. The ring connecting the bright spots indicates a polycrystalline nature. Furthermore, the spots from rGO9 are brighter than that of rGO6 and attributed to the better crystallinity of the former. Turbostatic stacking of graphene sheets is explicit where the bright spots are symmetric around the center maximum. The increased stacking is a result of the duration of reduction, thus increasing the polycrystallinity of the sample. Although the discussion of SAED patterns may correspond to graphite or graphite oxide, the changes due to reduction may be relevant in the case of the latter. To reveal the overall morphology of the reduced GO, SEM images of the rGO6 and rGO9 samples are shown in Fig. 1e and f. We observed large areas of continuous films apart from folded or wrinkled regions. Additional SEM images from rGO3, 6 and 9 are shown in Fig. S1 (ESI†). SEM images also revealed the presence of clusters of smaller particles. These particles are tentatively assigned to segregated amorphous carbon that forms during oxidation and subsequent reduction. We will see a signature of such type of carbon ${ }^{25}$ in the Raman spectral analyses.

Fig. 2 shows XRD patterns of films of GO and rGOx samples. The (002) reflection occurred at a $2 \theta$ value of $\sim 10.21^{\circ}$ corresponding to the interlayer distance $(d)$. The $d$ spacing values are calculated and found to be $\sim 0.84,0.79,0.78$ and $0.78 \mathrm{~nm}$ for the GO, rGO3, rGO6 and rGO9 films, respectively. As the reductiontime increases, the distance between the graphene layers decreases due to the removal of a fraction of oxygen containing functional groups. These functional groups hinder the van der Waals attraction forces between the layers. Our findings are consistent with the literature. ${ }^{2,8,13}$ Evidently, the $d$ value decreased from 0.84 to $0.79 \mathrm{~nm}$ and then to $0.78 \mathrm{~nm}$. Indeed the $d$ value of GO is very much dependent on the oxidation procedure, while that of rGO is dependent on the reduction procedure. In both cases trapped/intercalated molecules and/or atoms influence the experimental $d$ value. For example, trapped $\mathrm{H}_{2} \mathrm{O}$ increases the interlayer spacing. ${ }^{2,8,13}$ In the case of rGO6 and rGO9 films there is almost no change in the interlayer spacing, which is attributed to the intercalated water molecules, 


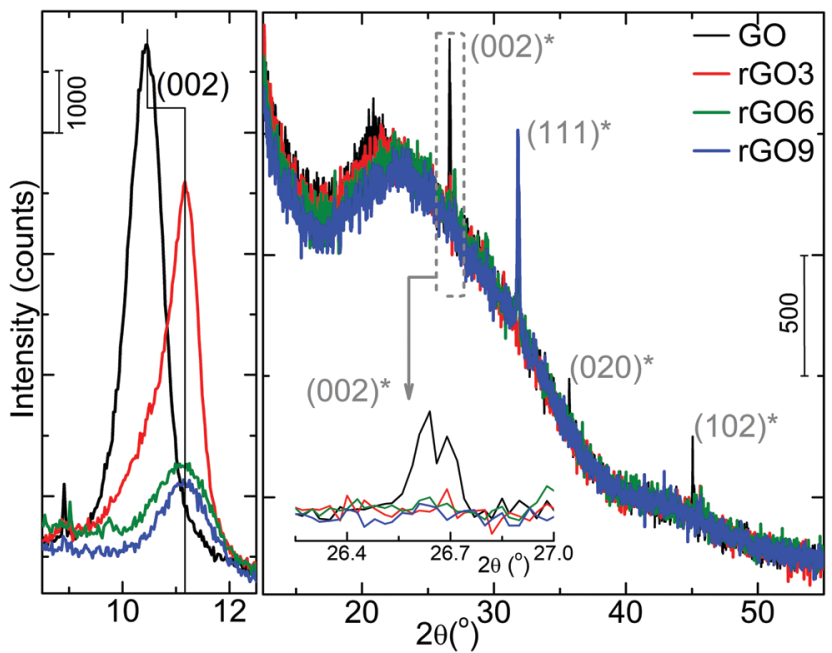

Fig. 2 XRD patterns of film samples of $\mathrm{GO}$ and after reduction for 3 hours (rGO3), 6 hours ( $r G O 6)$ and 9 hours (rGO9). Diffraction planes indicated with * belong to graphite/relatively less oxidized graphite.

despite further reduction. We will see that this attribution is corroborated by the density of functional groups from C1s spectra. The interlayer spacing is not comparable to that of graphite, suggesting that the graphene is still in the oxidized form after a $9 \mathrm{~h}$ photo-thermal reduction. Further analyses of the full width at half maximum (fwhm) of the (002) peak with Debye-Scherrer's equation revealed the mean dimensions of an ordered stack of graphene sheets normal to the diffracting plane. These values are determined to be $\sim 10.63,10.63,7.92$ and $7.92 \mathrm{~nm}$ for the GO, rGO3, rGO6 and rGO9 samples, respectively. The value of $10.63 \mathrm{~nm}$ corresponds to about 12 to 13 layers of oxidized graphene for the GO and rGO3 samples, while $7.92 \mathrm{~nm}$ corresponds to about 10 sheets, however, not considering the intercalated $\mathrm{H}_{2} \mathrm{O}$ in any case. Notably, the interlayer spacing is a vital factor, where the fluorescence quenching is achieved via improved $\pi-\pi$ stacking. ${ }^{9-11,15}$ We will evidence and discuss this effect on the fluorescence elaborately. The right panel in Fig. 2 depicts a higher $2 \theta$ region. We have observed $(002)^{*},(111)^{*}$, $(020)^{*}$ and $(102)^{*}$ reflections from the graphite lattice, where $(002)^{*}$ corresponds to the $\mathrm{AB}$ stacking of graphite/graphite oxide. $^{23}$ The $2 \theta$ region near the $(002)^{*}$ reflection (dotted grey box) is magnified and shown as an inset. The peaks corresponding to graphite might have occurred due to un-reacted or under-oxidized clusters, known as graphite oxide. ${ }^{23,24}$ The $(002)^{*}$ reflection is clearly visible only for the as-prepared GO sample. The intensity ratio is found to be $\left(I_{(002)} / I_{(002)^{*}}=\right) \sim 17$, where $I_{(002)}$ and $I_{(002)^{*}}$ correspond to the background subtracted intensities of the peaks from GO and graphite/graphite oxide, respectively. A nearly 17 times higher intensity from (002)/GO with reference to $(002)^{*} /$ graphite and/or -oxide is a clear indication of a very small contribution of the latter. Similarly, the relative intensities of the other reflections $\left((002)^{*},(020)^{*}\right.$ and $\left.(102)^{*}\right)$ are found to be rather lower for the reduced samples. On the other hand, (111)* depicted increased intensity upon reduction. This analysis clearly corroborates the discussion related to SAED patterns, where the fractions of graphite and/or graphite oxide

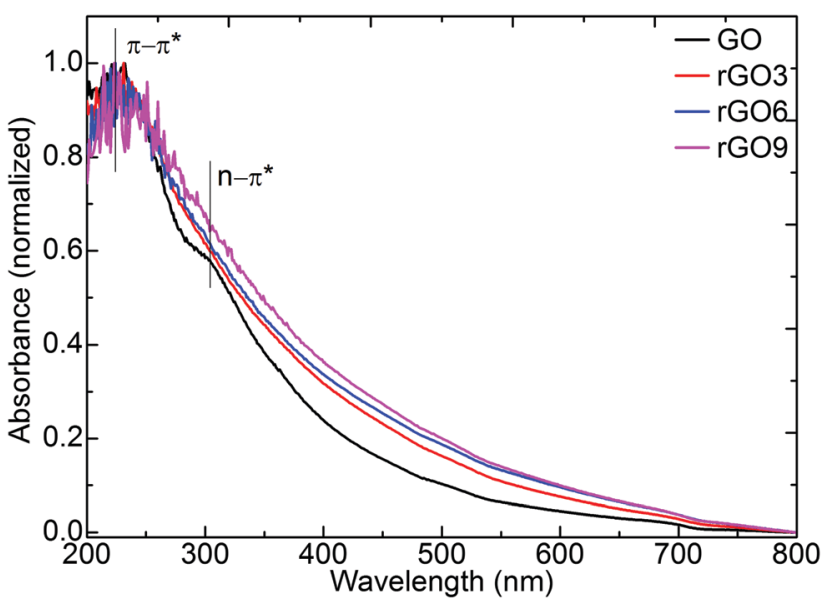

Fig. 3 UV-Vis absorption spectra from GO and after reduction for 3 hours (rGO3), 6 hours (rGO6) and 9 hours (rGO9).

are rather low. Hence in the present context, we have a majority fraction of exfoliated sheets due to severe oxidation. ${ }^{2,23,24}$

As mentioned earlier, GO is an oxo-functionalized form of graphene. By subjecting the GO to reduction, its electronic properties are altered where a transition from semiconductor to semimetal is expected. ${ }^{19}$ Fig. 3 shows the UV-Vis absorption spectra for aqueous dispersions of GO, rGO3, rGO6 and rGO9, where two main transitions are identified for all samples. Essentially, GO and rGOs depicted $\pi-\pi^{*}(\sim 250 \mathrm{~nm})$ and $\mathrm{n}-\pi^{*}$ $(\sim 310 \mathrm{~nm})$ transitions, where $\pi\left(\pi^{*}\right)$ stands for a bonding (anti-bonding) orbital. The main transition, $\pi-\pi^{*}$, is related to the common $\mathrm{C}=\mathrm{C}$ bonding in the $\mathrm{GO}$ and $\mathrm{rGOx}$ lattices. As the reduction time increases, a slight red shift is observed for the $\mathrm{C}=\mathrm{C}$ absorption band. This red shift may be attributed to the increased electron density and increased order on the graphene lattice, ${ }^{26}$ which stem from the removal of a fraction of oxygeneous functional groups. We will see that this is really the case in the context of C1s spectra. For the rGOx case the optical gap (HOMO and LUMO gap) is expected to be lower than that of GO. ${ }^{19}$ The intensity of the $n-\pi^{*}$ transition is relatively low, which is attributed to peroxide and/or epoxide linkages between GO layers. ${ }^{27}$ As a result, in the case of rGO samples this shoulder loses its intensity and spectral profile due to the depletion of oxygen bearing functional groups. ${ }^{3,27,28}$ We also note that absorption in the visible part of the spectra increased with reduction time. The absorption in the visible part of the spectra $(>400 \mathrm{~nm})$ occurred due to large conjugated domains (bathochromic shift of absorption bands from the benzene-like building block due to increased conjugation). The increased conjugation enhances the electron delocalization on the graphene lattice because of the reduction/removal of oxy-groups.

As outlined in the introduction, due to the applicability of graphene based materials, several methods are introduced for the synthesis of rGO from GO. The presence and density of oxygeneous functional groups on graphene depend on the method of preparation. In our earlier ${ }^{8}$ study, the $\mathrm{sp}^{2} \mathrm{C}=\mathrm{C}$ to oxidized-C ratios of the GO samples are $1.7: 1.0$. In contrast, here in this study, we find that the $\mathrm{O} / \mathrm{C}$ ranges from 2.2 to 1.86 
for GO through rGO9 (Fig. S2, ESI $\dagger$ ) from the XPS-survey (not shown here) and core-level spectroscopy. We did not detect any other chemical impurities in survey spectra (within the sensitivity), suggesting the contamination free GO and rGOx samples. At the end of 9 hour reduction a total of 4 at\% oxygen is removed with respect to that of GO. The resulting rGO is unique regarding its surface properties such as functionalization and defect density and distribution, which typically depend on the experimental conditions. It is also notable that the reduction may not completely recover the graphene due to the associated defects. ${ }^{17}$

C1s core-level spectra with the spectral deconvolution of as prepared GO and its reduced counterparts are depicted in Fig. 4a. The fractional contribution of each of the functional groups is also indicated in Fig. 4b. These groups include RCOO or $-\mathrm{COOH}, \mathrm{RC}=\mathrm{O}$ or $\mathrm{HC}=\mathrm{O}, \mathrm{C}-\mathrm{O}-\mathrm{C}$ and $\mathrm{C}-\mathrm{OH} .^{6,8,17}$ Clearly, it can be seen that the total oxygen fraction decreases with increasing reduction. In the case of the reduced samples, there is an apparent red shift of electron binding energy for all functional
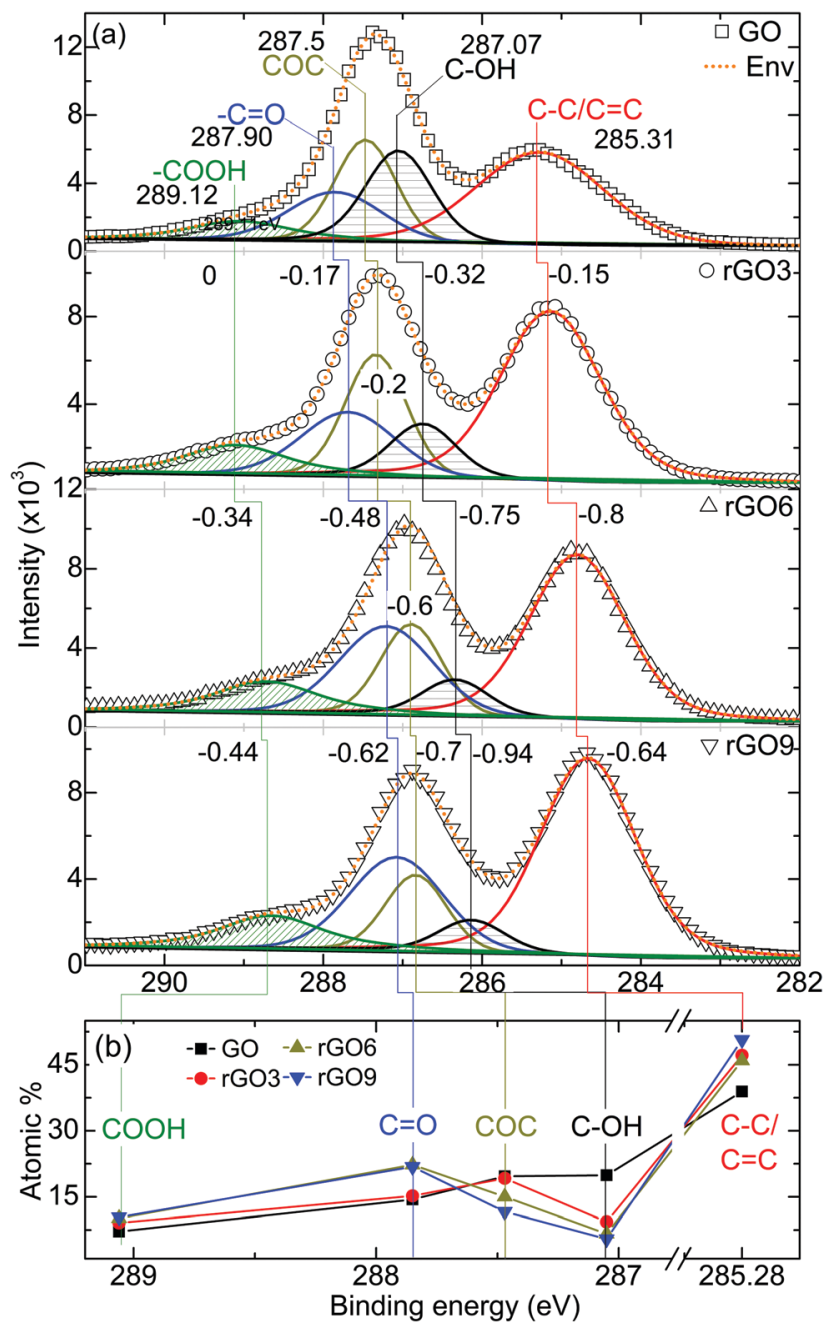

Fig. 4 (a) $\mathrm{C} 1 \mathrm{~s}$ spectra of $\mathrm{GO}$ and after reduction for 3 hours ( $\mathrm{rGO}$ ), 6 hours ( $r G O 6$ ) and 9 hours (rGO9), and (b) atomic percentage of each of the functional groups is plotted for all the samples. Functional groups are annotated on the spectra with spectral locations and relative shifts. Connecting lines in part (b) serve as guides to the eye. groups due to the reduced total oxygen content. The spectral shifts are identified in the figure, which are attributed to the (A) decreased density of oxygeneous functional groups and (B) increased delocalization of electrons. Since oxygen is a relatively electro-negative atom, its functional groups depict an electronwithdrawing nature. As a result of point (A), the fraction of withdrawn electrons from graphene also decreases; hence, the binding energy of the photoelectron decreases.

For the as prepared GO sample, the functional groups are listed in decreasing order of percentage occurrence in the following: $-\mathrm{OH}, \mathrm{C}-\mathrm{O}-\mathrm{C}, \mathrm{C}=\mathrm{O}$ and $-\mathrm{COOH}$. There is a sharp increase of the $\mathrm{C}-\mathrm{C} / \mathrm{C}=\mathrm{C}$ component after the first 3 hour reduction. As the reduction progresses, the $\mathrm{C}-\mathrm{C} / \mathrm{C}=\mathrm{C}$ component is found to increase, however, not as much as that of first 3 hour reduction. Clearly, we did not reach the saturation in reduction as also observed in the context of interlayer spacing/ $\mathrm{XRD}$ analyses. For the GO sample, the $-\mathrm{OH}$ functional group is predominant, which loses its intensity relatively more rapidly within the first 3 hours of photo-thermal reduction. Under suitable illumination the lone electron pair of the oxygen atom in the $-\mathrm{OH}$ group includes repulsive singlet and triplet terms. Similarly, the $\mathrm{C}-\mathrm{O}$ bond exhibits the same repulsive terms. Hence, for the pure photo-chemical process the detachment of $-\mathrm{OH}$ is feasible. However, we also should acknowledge the fact that we supply heat to the system in the presence of $\mathrm{H}_{2} \mathrm{O}$, which has its own implications on the reduction process, as described earlier. ${ }^{12}$ For the rGO6 and rGO9 samples, the $-\mathrm{OH}$ contribution still decreases, however, at a lower rate. Interestingly, this observation can be juxtaposed with the changes to the fraction of $\mathrm{C}-\mathrm{C} / \mathrm{C}=\mathrm{C}$. The fraction of $\mathrm{C}-\mathrm{C} / \mathrm{C}=\mathrm{C}$ appeared to have a sharp rise for rGO3 and then increased at a slower rate as the degree of reduction increases. We note a nominal decrease in the fraction of $\mathrm{C}-\mathrm{O}-\mathrm{C}$ functional groups for rGO3. However, from $6 \mathrm{~h}$ to $9 \mathrm{~h}$ reduction there is a notable loss in the fractional contribution. Interestingly, the case with the $-\mathrm{C}=\mathrm{O}$ is different from those of the other functional groups. There is a nominal increase of $-\mathrm{C}=\mathrm{O}$ in rGO3, while the rGO6 and rGO9 samples depicted almost the same fractional level. This suggests some sort of saturation in the formation of $-\mathrm{C}=\mathrm{O}$.

For other functional groups, the changes to $\mathrm{C}-\mathrm{O}-\mathrm{C}$ are distinct. A nominal decrease is observed for rGO3 when compared to that of the GO sample. Further increase in the reduction decreased its fraction. In the context of the $-\mathrm{COOH}$ group there is a consistent, however, nominal increase of its fraction. During the photo-thermal reduction, the $-\mathrm{COOH}, \mathrm{C}-\mathrm{O}-\mathrm{C}$ and $\mathrm{C}-\mathrm{OH}$ groups were proposed to take part in reduction, leading to the formation of a defect carbon and/or $\mathrm{CO}_{2}$ apart from a GO${ }^{\bullet}$ radical. This radical takes part further in the reaction with other carbon atoms rebuilding $\mathrm{sp}^{2}$ clusters or disordered units (see Box S1, ESI, $\dagger$ and references therein). In the present context, we observed an increase of the fractions of $\mathrm{sp}^{2}$ carbon, $-\mathrm{C}=\mathrm{O}$ and $-\mathrm{COOH}$. Despite this regeneration, a significant drop in the oxygen fraction is still recorded. This observation is convincing, where the formation of $\mathrm{GO}^{\bullet}$ radicals is inevitable and might lead to the formation of $-\mathrm{C}=\mathrm{O}$ and $-\mathrm{COOH}$ groups. Although this finding is rather interesting, further studies are essential 
(which are in progress) to disentangle the actual mechanism in addition to those suggested in Box S1 (ESI $\dagger$ ).

FTIR spectra depicted spectral features corresponding to the $\mathrm{C}=\mathrm{O}\left(1630 \mathrm{~cm}^{-1}\right), \mathrm{OH}, \mathrm{CH}\left(2921\right.$ and $\left.2848 \mathrm{~cm}^{-1}\right)$ and $\mathrm{C}-\mathrm{O}$ $\left(1430 \mathrm{~cm}^{-1}\right.$ ) functional groups (Fig. S5, ESI $\dagger$ ). This is consistent with the observation from photoelectron spectra. The frequency response from the hydroxyl group contains multiple components; however, it is centred at $\sim 3440 \mathrm{~cm}^{-1}$ with two clear shoulders on either side of the centre maximum ( 3250 and $3562 \mathrm{~cm}^{-1}$ ). $3562 \mathrm{~cm}^{-1}$ corresponds to terminal hydrogen bonds, while $3440 \mathrm{~cm}^{-1}$ and $3250 \mathrm{~cm}^{-1}$ are attributed to polymeric-type hydrogen bonds. We parameterized the $\mathrm{OH}$ response region to fit three peaks for all samples. The changes due to the reduction were quantified with respect to those of GO and are tabulated in Table S1 (ESI $\dagger$ ). Indeed the $\mathrm{COOH}$ group contributes to the vibrational bands corresponding to the $\mathrm{OH}$ and $\mathrm{C}=\mathrm{O}$ regions. On the other hand, the presence of individual $\mathrm{C}=\mathrm{O}, \mathrm{OH}$, and $\mathrm{COC}$ groups in the GO and rGOx samples is notable. Although, in principle, the resonance frequency of $\mathrm{C}=\mathrm{O}$ from $\mathrm{COOH}$ might be different from that of the individual $\mathrm{C}=\mathrm{O}$ group, due to the presence of intercalated $\mathrm{H}_{2} \mathrm{O}$ and the associative behavior of graphene oxide sheets it is difficult to disentangle the number of peaks, their spectral position, and their fractional contribution. Indeed, this argument is also applicable to the other oxygeneous functional groups. Hence the results obtained from FTIR are cumulative (bulk) effects from various functional groups. Upon reduction the fractional contribution from the oxygeneous functional groups decreases (Table S1, ESI $\dagger$ ). Surprisingly, as the reduction increases we note an increase in the fraction of $\mathrm{C}-\mathrm{H}$ groups, occurring from aliphatic or allylic carbon. This is well consistent with our earlier comment that as the reduction takes place there can be still oxidation reactions mediated by radicals.

Raman spectra from GO dispersion and film samples within $\sim 1000-1900 \mathrm{~cm}^{-1}$ are shown in Fig. 5a and b, respectively. We also deconvoluted these spectra to unveil the contributions of

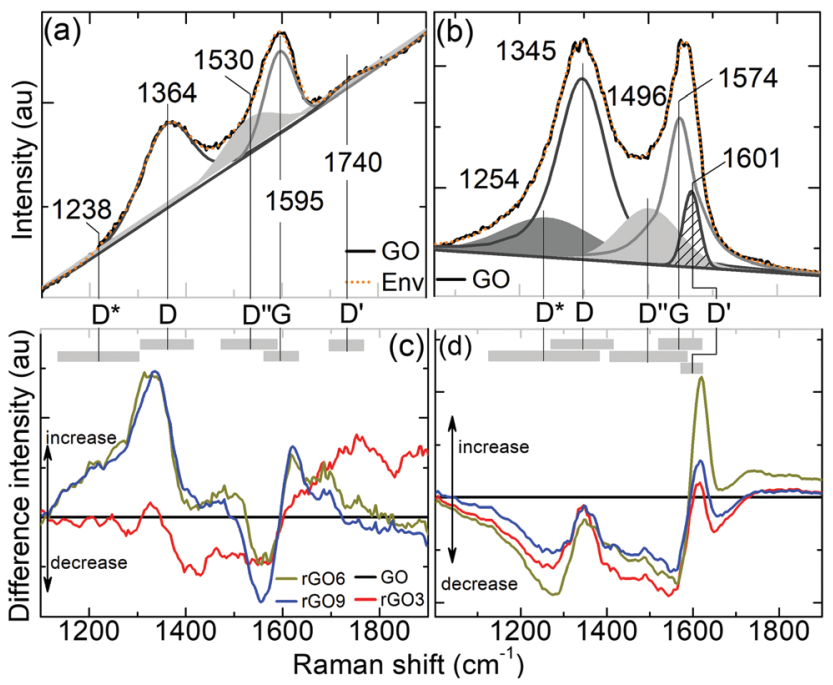

Fig. 5 Raman spectra from the GO (a) dispersion and (b) film samples. The intensity differences for the reduced samples ( $r G O x-G O)$ are compared with reference to the GO (c) dispersion and (d) film. Grey rectangles on parts (c) and (d) correspond to the fwhm of the vibrational band. individual bands of different origins. ${ }^{25}$ In this deconvolution 4-bands are defect mediated $\left(\mathrm{D}^{*}, \mathrm{D}, \mathrm{D}^{\prime \prime}\right.$ and $\left.\mathrm{D}^{\prime}\right)$, while the 5 th one is the well known $\mathrm{G}$ band. ${ }^{25} \mathrm{D}^{*}$ and $\mathrm{D}^{\prime \prime}$ are in fact poorly understood modes of graphene/graphene oxide; for instance, see citations 32, 34-36, 39 and 40 in ref. 25. Hence it is important to assign each defect to the corresponding process, especially when dispersion and film cases are juxtaposed. On the other hand, the degree of doping and information related to the lattice-strain can also be obtained from Raman spectroscopy. ${ }^{29}$

The spectral location of each of the deconvoluted bands is annotated in Fig. 5a and b. Clearly, except for $\mathrm{D}^{*}$, all the other bands depicted a red shift upon removal of dispersant. In the following, we discussed each band with reference to the spectral shift due to the presence of the dispersant. To start with, the $\mathrm{D}^{*}$ (or $\mathrm{T}$ ) band is attributed to the $\mathrm{sp}^{3}$ hybridized bonds of $\mathrm{C}-\mathrm{C}$ or $\mathrm{C}-\mathrm{O}$ at the edge. This band occurred at $1238 \mathrm{~cm}^{-1}$ for GO dispersion, which is blue shifted in the film counterpart. During the formation of the film, conjugated domains would stack, causing a strain on the $\mathrm{C}-\mathrm{C}$ or $\mathrm{C}-\mathrm{O}$ bands, in contrast to dispersion. The $\mathrm{D}$ band generally occurs due to defects, which depicts a shift towards a low frequency upon film formation. In the case of the dispersion samples, these defects are under the influence of hydrogen bonds from the dispersant. This might have caused the Raman shift to occur at a higher frequency in dispersion. The $\mathrm{D}^{\prime \prime}$ band represents amorphous phases in the structure. The shift occurring at higher frequency in dispersion is clear evidence of the interaction of $\mathrm{H}_{2} \mathrm{O}$ molecules on the structure. The hydrophobic nature of the amorphous carbon is subjected to confinement causing a red shift upon film formation.

Furthermore, we find a significant red shift of the $G$ band upon transformation of dispersion into film. The softening of the $\mathrm{G}$ band can be attributed to the decreased doping level on the graphene. ${ }^{30}$ In the case of dispersion, the dispersant forms hydrogen bonds with the oxygeneous functional groups on the graphene, where the $\mathrm{O}^{-\delta} \mathrm{S}$ of oxy-groups are screened by $\mathrm{H}^{+\delta} \mathrm{S}$ of $\mathrm{H}_{2} \mathrm{O}$. This relatively increases the electron density on the graphene. Once the dispersant is removed the oxygeneous functional groups decrease the electron density on the graphene due their inherent electron withdrawing nature. The $\mathrm{D}^{\prime}$ band requires a defect (similar to that of the $\mathrm{D}$ band) and attributed to point phonons (magnitude of the phonon wave vector is equal to zero). ${ }^{31}$ The presence of the $\mathrm{D}^{\prime}$ band is related to 5 and/or 7 member rings. The $\mathrm{D}^{\prime}$ band occurred at 1740 and $1601 \mathrm{~cm}^{-1}$ for the dispersion and film GO samples, respectively. It is nontrivial to determine the cause of shifts of such magnitude. Further insight may be required from the computation, as the electronic structures of GO and or rGO are rather complex. However, this experimental finding would be a useful input for further studies. Contextually, in our earlier study we observed hydrogen bonding between $1^{\circ}$ or $2^{\circ}$ alcohols and the functional groups on the surfaces of GO films. ${ }^{8}$ We observed shifts in the spectral position of the $\mathrm{G}$ band as high as $12 \mathrm{~cm}^{-1} .8$

Having discussed the origin and related shifts of each of the bands, to reveal the effect of reduction clearly we evaluated the differences in the Raman response (rGOx-GO) for both the 
dispersion and film cases, which are shown in Fig. 5c and d, respectively. Changes will be discussed with reference to that of GO, which is considered as a baseline with zero intensity. Grey boxes on parts (c) and (d) indicate the fwhm of the bands corresponding to that of GO in dispersion and film forms. ${ }^{32}$ At first glance, we observed a stark contrast between the trends for dispersion and film cases. Dispersions of rGO6 and rGO9 have shown similar trends in contrast to that of rGO3. Interestingly, film samples depicted similar trends apart from the magnitude especially in the $\mathrm{D}^{\prime}$-band region.

Going into the details, the intensity of $\mathrm{D}^{*}$ is almost invariant for rGO3. This region is more intense for the rGO6 and rGO9 dispersions. It appears to be the case that the presence of $\mathrm{H}_{2} \mathrm{O}$ in fact enhanced the relative intensity. On the other hand, an increase in the $\mathrm{D}^{*}$ band suggests that the photo-thermal reduction selectively reduces the functional groups; however, in this process a transformation of functional groups is also observed from XPS. During the transformation vacancy or disordered ring formations are expected, which increases the intensity of the $\mathrm{D}^{*}$ band. Interestingly, for film samples the intensity is found to decrease, where the $\mathrm{sp}^{2}$ stacking is predominant. As the degree of reduction increases, there is a net loss of the density of $\mathrm{C}-\mathrm{O}$, as evidenced from the films. For the $\mathrm{D}$ band region, almost the same level of intensity is observed for rGO6 and rGO9 in contrast to that of the rGO3 film. The D band might also occur from defects at grain boundaries, which would be predominant in the dispersion. Essentially, the lattice discontinuity is enhanced via $\mathrm{H}_{2} \mathrm{O}$ intercalation.

We acknowledge the fact that the domain size of $\mathrm{sp}^{2}$ carbons does not change from dispersion to film. Interestingly, the intensity of the D band is almost invariant for rGOx films, however, lower than that of from the GO film. This can be attributed to the associative behaviour of $\mathrm{sp}^{2}$ domains. $\mathrm{D}^{\prime \prime}$, on the other hand, lost its intensity, however, similar to those of the film samples. The intensity of the $\mathrm{D}^{\prime \prime}$ band did not change from dispersion to film, and hence, no severe intensity changes are expected. The $\mathrm{G}$ and $\mathrm{D}^{\prime}$ bands overlap in the frequency domain. Similar to the case of the D-band, the associative behaviour of $\mathrm{sp}^{2}$ domains selectively enhanced its intensity. A stark contrast between the trends of the dispersion and film forms hinges on the fact that the hydrogen bonds of functional groups with $\mathrm{H}_{2} \mathrm{O}$. In the film-form, at least 10 graphene oxide layers are stacked (see $\mathrm{XRD}$ results), however, mediated by the associative behaviour of $\mathrm{sp}^{2}$ and the functional groups.

Oxidation of graphene implants various functional groups such as $-\mathrm{COOH},-\mathrm{C}=\mathrm{O},-\mathrm{C}-\mathrm{O}-\mathrm{C}-$ and $-\mathrm{OH}$ as evidenced from core-level XPS. ${ }^{8}$ These functional groups form hydrogen bonds with the dispersant, which are presumably the primary reason for the stabilization of a water-dispersion. We will see the effect of hydrogen bonds and varying degrees of oxidation on the fluorescence properties where we juxtaposed the film and dispersion cases (Fig. 6). Fig. 6a and b show the fluorescence spectra from the dispersion and film samples, respectively, for a selected excitation wavelength of $345 \mathrm{~nm}$. At first glance, explicit differences were evident in the fluorescence spectra where the changes in the dispersion are in contrast to its film counterparts

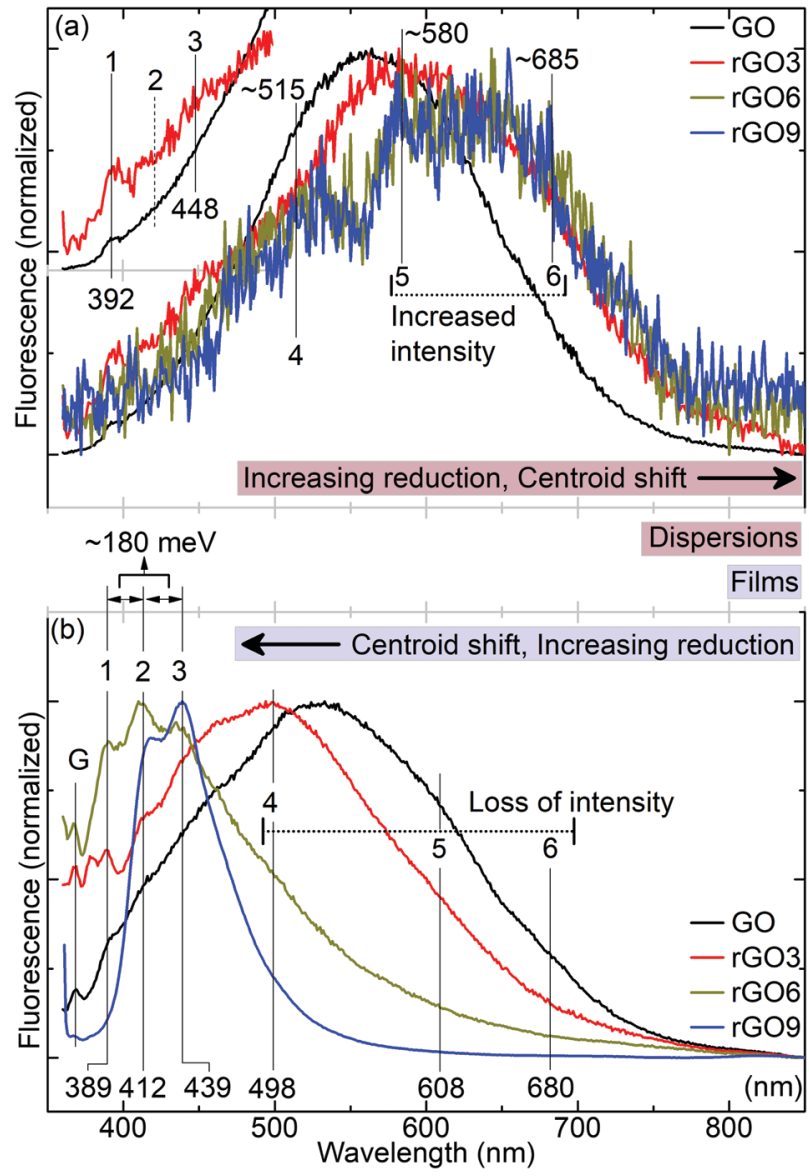

Fig. 6 (a) Fluorescence spectra from dispersion samples of the as prepared GO and after reduction for 3 hours (rGO3), 6 hours (rGO6) and 9 hours (rGO9). (b) Film samples of the as prepared GO and after reduction for 3 hours (rGO3), 6 hours (rGO6) and 9 hours ( $r G O 9$ ). Excitation wavelength = $345 \mathrm{~nm}$ for each case.

with increasing degree of reduction. Although the fluorescence from dispersions, GO and rGO3 films appears to be single broad emission, a closer analysis reveals the presence of multiple components ( 1 through 6 ). The spectral positions are annotated in Fig. 6 for the dispersions and films as well. The inset of Fig. $6 \mathrm{a}$ shows emission in the 350 to $500 \mathrm{~nm}$ region for the GO and rGO3 dispersions. As the degree of reduction increases, the emission intensities from the dispersions are subdued significantly (Fig. 6a). On the other hand, in the case of the films, the fluorescencemaximum occurred at $525 \mathrm{~nm}$, which blue shifted upon increasing the degree of reduction. This is in contrast to those of the dispersions, which have shown a red shift from $560 \mathrm{~nm}$ to $625 \mathrm{~nm}$, upon reduction. Spectral shift versus centroid-shift should be identified cautiously unless the spectral shapes are explicitly distinguishable. From Fig. 6a, upon reduction, we find that components 5 and 6 depicted enhanced intensity when compared to those of peaks 1 to 4 . It is in fact a shift of the centroid. Although peak 2 is not well resolved in the case of dispersions, the spectral location is tentatively indicated based on the observation from film counterparts. We will see that this attribution is indeed true when we discuss the spectra from the film samples. 
It is interesting to note a Raman active mode (label G on Fig. 6b) on the fluorescence spectra that appeared for all films with an equivalent shift of $0.224 \mathrm{eV}$ or $1807 \mathrm{~cm}^{-1}$. The G-band typically depicts strong dispersion, which is generally observed around $1600 \mathrm{~cm}^{-1}$. Hence, for $345 \mathrm{~nm}$ excitation, a shift of about $200 \mathrm{~cm}^{-1}$ is consistent with the literature. ${ }^{33}$ Notably, the rate of dispersion is proportional to the degree of disorder. ${ }^{33}$ Among the film samples, GO depicted a broad spectrum similar to that of its dispersion counterpart with various components as annotated on the spectra ( 1 through 6$)$. We will discuss the origin of these six peaks and the mutually contrasting behaviours of the dispersion and film samples in the following. Peaks 1 and 3 (inset of Fig. 6a) occur due to localized $\mathrm{sp}^{2}$ clusters of four or more 6-membered rings. Identifying the size of the cluster is not trivial due to the complexity of GO or rGO structures and their associative behaviour in solvent and films, where they tend to form clusters mediated by van der Waals attractive forces. We observed severe changes from samples with higher degrees of reduction (rGO6 and rGO9). As the degree of reduction increases, the fwhm values of peaks 1 , 2 and 3 decreased substantially and systematically. The decreased fwhm indicates smaller clusters, where the electrons relatively more localized. Convincingly, as we reduce the GO, we decrease oxygeneous functional groups while forming $\mathrm{sp}^{2}$ clusters. The size of the cluster decreases as the reduction increases with further cleaving of graphene layer. However, we find that this is not always the case with photo-thermal reduction. Depending on the degree of oxidation and functionality, it is possible to control the domain size (results not shown here). More interestingly, peaks 1 and 2, and 2 and 3 are separated by $\sim 180 \mathrm{meV}$, which correspond to the CC stretch mode, and the spectral features resemble the emission spectra of anthracene and tetracene-like molecular clusters. ${ }^{34}$ Finding the emission that can be attributed to molecular features supports the argument of the formation of small sized $\mathrm{sp}^{2}$ domains and their contribution to the fluorescence spectrum. ${ }^{6}$ Peaks 4,5 and 6 are also attributed to $\mathrm{sp}^{2}$ molecular clusters, however, with relatively larger cluster size. This higher cluster size red shifted the emission spectrum due to the electron delocalization. We explicitly observe a CC stretch vibrational spacing of $\sim 180 \mathrm{meV}$ between peaks 3 and 4 (results not shown here).

Although the emission from GO is due to $\mathrm{sp}^{2}$ clusters, it is also notable that the oxygeneous functional groups have crucial role to play in the relative intensities of each of the bands. The GO film depicts a broad spectrum as in the case of dispersion (Fig. S3, ESI $\dagger$ ), however, with considerable changes to the spectral features. Once the solvent evaporates, the centroid of the emission maximum shifts from $565 \mathrm{~nm}$ to $525 \mathrm{~nm}$. Apparently, in the case of GO the presence of $\mathrm{H}_{2} \mathrm{O}$ subdues the intensities of peaks 1 through 4 while enhancing 5 and 6 . Essentially, we are dealing with a mixture of conjugated polycyclic and aromatic compounds functionalized with oxygeneous functional groups. These functional groups form hydrogen bonds with the dispersant, $\mathrm{H}_{2} \mathrm{O}$. The presence of such bonds will slightly increase the electron density on the $\mathrm{sp}^{2}$ clusters as the electronegative oxygen $\left(\mathrm{O}^{-\delta}\right)$ atom from the oxy-group is partially screened by $\mathrm{H}^{+\delta}$ ions from $\mathrm{H}_{2} \mathrm{O}$. On the other hand, the bond strength depends on the electronegative character of the oxygeneous functional group ( $\mathrm{COOH}, \mathrm{RC}=\mathrm{O}$ or $\mathrm{HC}=\mathrm{O}, \mathrm{C}-\mathrm{O}-\mathrm{C}$ and $\mathrm{C}-\mathrm{OH}$ ), see citations 26 and 28 in ref. 35. For example, significant changes to the emission spectra from GO were reported as a function of $\mathrm{pH}^{16}$ Increasing the duration of reduction increases the density of smaller $\mathrm{sp}^{2}$ domains, which are attributed to peaks $1-3$, while at the same time reduction decreases the larger domains, giving rise to peaks 4 through 6 . We cannot exclude the presence of oxygeneous functional groups on $\mathrm{sp}^{2}$ domains of any size. In the dispersions, as the degree of reduction increases, the density of smaller $\mathrm{sp}^{2}$ domains also increases. Since they have functional groups on the lattice, they are under the influence of $\mathrm{H}_{2} \mathrm{O}$, which suppresses the emission intensity. On the other hand, larger $\mathrm{sp}^{2}$ domains are less influenced by the effect of hydrogen bonds; however, the emission decreases due to the increased fragmentation. Since we observe emission from peaks 4-6 in the dispersion, relatively large $\mathrm{sp}^{2}$ domains are in existence. As the dispersant is removed, smaller $\mathrm{sp}^{2}$ domains depict clear lines of emission (peaks 1-3). In the case of larger $\mathrm{sp}^{2}$-domains the associative behaviour depicts enhanced quenching efficiency. Due to the reduced degree of oxidation, the layers come closer and hence very efficient quenching of fluorescence via $\pi-\pi$ interactions. ${ }^{9,15}$ Indeed we observed a decrease in the interlayer spacing from the XRD measurements on the film samples.

Our findings are in stark contrast to a study by Chien et al. ${ }^{6}$ where the luminescence is attributed to having two components and increasing reduction caused a blue shift. On the other hand, no information is given about the luminescence from film counterparts in ref. 6 . Galande et al. ${ }^{16}$ observed 5 components in the fluorescence spectra for $440 \mathrm{~nm}$ excitation. In this study, as the $\mathrm{pH}$ becomes basic, the low energy components lose their intensity. The emission is interpreted as occurring from quasimolecular species where the fluorescence is influenced by $\mathrm{pH}$ via the $\mathrm{COOH}$ group. However, notably, hydrogen bonding may occur via other oxygeneous functional groups, see citations 26 and 28 in ref. 35 . In such a scenario, we expect a cumulative effect of the hydrogen bonds on the electronic structure, where the effect can be factorized based on the density of functional groups from XPS (Fig. 4b). Importantly, the degree of oxidation and the distribution of functional groups play a key role in determining the emission properties, especially the strength of the interaction with the dispersant depends on the functional group, as mentioned earlier. That is, negative charge due to the presence of $\mathrm{H}_{2} \mathrm{O}$ is localized on one oxygen atom in the case of $\mathrm{R}-\mathrm{OH}$, but on two oxygen atoms in the case of $\mathrm{R}-\mathrm{COOH}$ forming $\mathrm{H}_{3} \mathrm{O}^{+}$. This argument can be further extended to 'inductive' effects due to other functional groups. Furthermore, Galande $e t$ al. ${ }^{16}$ reported red-shifted spectra under acidic conditions and ruled out the possibility of any aggregation. No data are available for the film cases in ref. 16 which may unveil the effect of aggregation and confinement. Hence it is important to consider spectral shift $v s$. centroid shift where we find strong evidence of subdued intensity due to aggregation ${ }^{9-11,15}$ compared to any spectral shift upon film formation. This is also the 
case with Chien et al.'s study. ${ }^{6}$ Furthermore, multiple components in the fluorescence spectra can be seen in a study by Rani et $a l . ;^{22}$ however, no data from dispersions are available. See our earlier review article ${ }^{17}$ for various other studies.

In the normalized spectra (Fig. 6), it appears that the wavelength-integrated intensity decreases with increasing reduction at least in the context of dispersions. We calculated these values with respect to GO for $345 \mathrm{~nm}$ illumination for the dispersion and film samples. The calculated values are 9.2, 2.2 and $1.7 \mathrm{~nm}$ cps for the rGO3, rGO6 and rGO9 dispersions, respectively. In the case of the dispersion samples there is a consistent decrease in the yield, as discussed earlier. However, for the film samples, these values are 23.8, 12.5 and $59.2 \mathrm{~nm}$ cps for rGO3, rGO6 and rGO9, respectively. In this case, with reference to the GO film, the efficiency gradually decreases until 6 hours of reduction. For the rGO9 sample, a significant increase in the emission intensity is due to the highly fluorescent molecular species of relatively higher fraction than that of rGO6. The changes to the density of molecular fragments are clearly apparent from the fluorescence spectra where we observe the components 4 and 5 from rGO6. The emission yields from the dispersions and films depend on the density of functional groups under the influence of hydrogen bonds and the selfquenching phenomenon, respectively. Nevertheless, in the application point of view, rGOx may be employed as a fluorescence quencher and an efficient energy transfer medium where lower fluorescence is required.

To understand the emission properties further we performed excitation dependent fluorescence measurements on all samples. Exemplary responses from selected GO, rGO3 dispersions and films of GO, rGO3, rGO6 and rGO9 are shown in Fig. 7. Emission intensities are normalized and shown in a false color scale with reference to excitation wavelengths. Also, refer to Fig. S4 (ESI $\dagger$ ) for the luminescence spectra for selected excitation wavelengths. As discussed in the context of Fig. 6 we observed Raman response and annotated it on the spectra. The intensity of the Raman feature is significant for the rGO6 and rGO9 samples. As the excitation energy decreases, the intensity of the emission also decreases for all samples. On the other hand, the emission shifts to a lower energy side where the spectral feature of peak 6 remains persistent. The relative intensity of this feature decreases with increasing degree of reduction. This is attributed to an increased quenching effect via $\pi-\pi$ interactions. It is notable that the larger $\mathrm{sp}^{2}$ domains have smaller optical gaps, where a higher wavelength $(619 \mathrm{~nm})$ can still produce electron-hole pairs. This is equivalent to an optical gap of about $2 \mathrm{eV}$. Interestingly, in the case of the rGO6 and rGO9 samples, the molecular feature appeared for excitations around $400 \mathrm{~nm}$, which we believe is due to resonance excitation. This in fact supports the argument that emission from graphene oxide is due to localized $\mathrm{sp}^{2}$ domains. In a realistic scenario, chemically prepared GO/rGO depicts a varying optical gap, which ranges from $\sim 3.5$ to $2 \mathrm{eV}$ (estimated from the excitation energy without considering any Stokes shift). It is also non-trivial to control the optical gap of a single graphene layer of larger dimensions. Essentially, each graphene sheet consists of domains of various

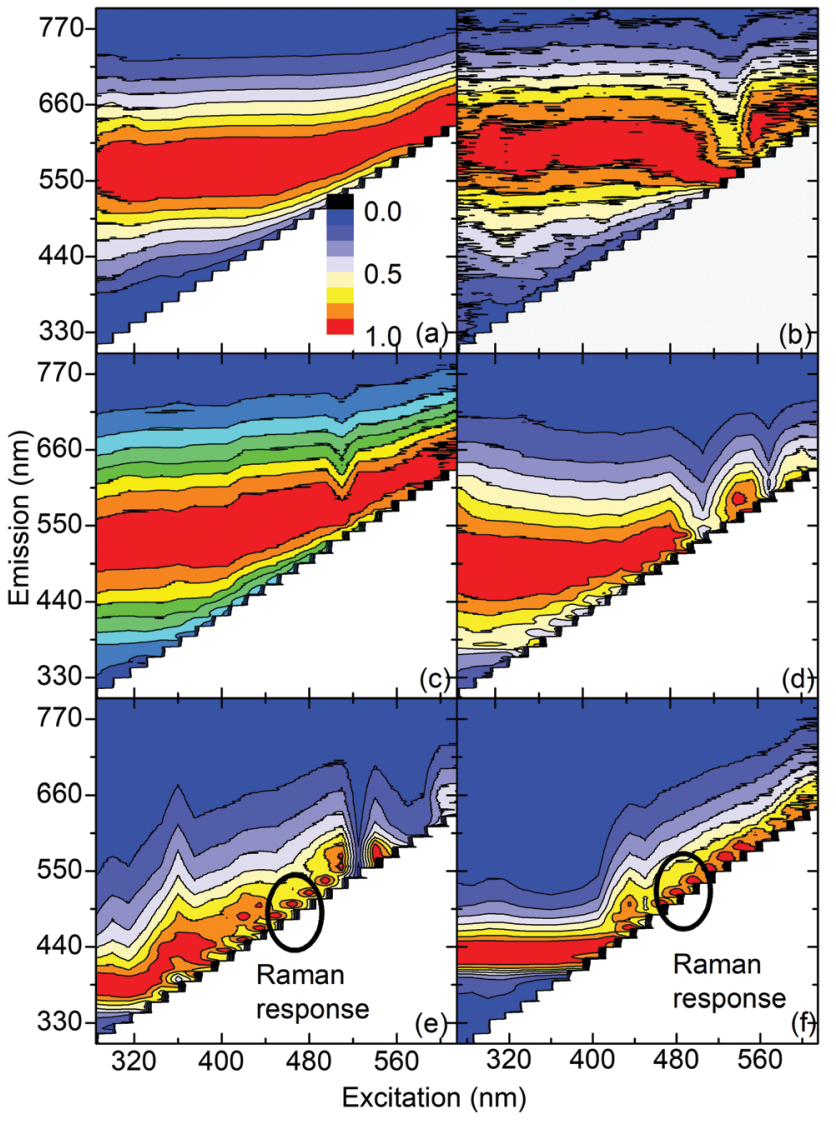

Fig. 7 Excitation-emission mapping from dispersions of (a) $\mathrm{GO}$ and (b) rGO3 and films of (c) GO, (d) rGO3, (e) rGO6 and (f) rGO9. The emission intensity is normalized and shown in a false colour scale.

optical gaps depending on the confinement via broken bonds and oxygeneous functional groups. Furthermore, the spectral positions of peaks 1 through 6 remain unchanged for all excitation energies, supporting our argument. Strong evidence of the influence of hydrogen bonds together with the self-associative behaviour is a clear indication of emission from $\mathrm{sp}^{2}$ clusters. Also note that an alternative mechanism for fluorescence involves the $\mathrm{O} 2 \mathrm{p}$ orbital where the conduction band of the localized $\mathrm{sp}^{2}$ domains can be assigned to the $\pi^{*}$ orbitals, while the VB changes from the $\pi$ to $\mathrm{O} 2 \mathrm{p}$ orbitals. See citations 15 , 36 and 39 in ref. 17. Having said that, the role of $\mathrm{O} 2 \mathrm{p}$ needs further investigation as the reduction procedure forms various by-products. ${ }^{17}$

\section{Conclusions}

Microscopy observations revealed that GO films consist of wrinkles and folded graphene oxide sheets, supporting a successful exfoliation of graphite. TEM analysis showed that as reduction takes place more $\mathrm{sp}^{2}$ domains are stacked. The associative behaviour of the GO sheets led to the stacking when the density of oxygeneous groups decreased. SAED patterns indicated the presence of graphite oxide. The $d$ value decreased for the first $6 \mathrm{~h}$ reduction, which then remained nominally the same. 
The invariant $d$ for higher reduction is due to the intercalation of water molecules, as we note a decrease in the total oxygen content from XPS. Furthermore, the crystallite size of $\mathrm{sp}^{2}$ regions was constant for the first $3 \mathrm{~h}$, then decreased $\sim 30 \%$ for $6 \mathrm{~h}$ and remained constant for $9 \mathrm{~h}$ reduction. Optical absorption spectra suggested depletion of oxygeneous groups and an increased density of $\mathrm{sp}^{2}$ domains upon reduction. An effective red shift in the binding energy of electrons from C1s is observed. This is indicative of the loss of oxygen bearing functional groups, and the retrieval of electron density on the carbon lattice. Interestingly, $\mathrm{C}-\mathrm{OH}$ remained the most influenced group during reduction. Furthermore, increase of $-\mathrm{C}=\mathrm{O}$ and $-\mathrm{COOH}$ fractions are attributed to radical $\left(\mathrm{GO}^{\bullet}\right)$ mediated reactions. Interestingly, film samples depicted similar Raman spectral profiles, however, with varying intensities. The longer the reduction time, the more the lattice is prone to vacancies, disordered ring formation related defects, and/or stacking disorder. Mainly, the softening of the G-band in the films is due to the decreased charge density. Fluorescence from GO is due to localized $\mathrm{sp}^{2}$ domains; however, it is influenced by the presence of $\mathrm{H}$ bonds and/or associative behaviour in the case of dispersions and films, respectively. We find that the fluorescence consists of 6 components. By increasing the reduction time the relative intensities of the various components changed significantly. This is rather clear and quite systematic for film samples where the lower energy components extinguish, while the higher energy peaks evolve and become sharper. The H-bonds suppress the emission from smaller $\mathrm{sp}^{2}$ domains, while the large domains remain almost unaffected. We also observed internal vibrational relaxation with a separation of $180 \mathrm{meV}$ for rGO6 and rGO9 samples, a clear indication of the molecular origin of fluorescence from $\mathrm{sp}^{2}$ domains.

\section{Conflicts of interest}

There are no conflicts to declare.

\section{Acknowledgements}

SV would like to thank TUBITAK BIDEB 2221 Fellowships for Visiting Scientists and Scientists on Sabbatical Leave for a postdoctoral fellowship, 2014.

\section{Notes and references}

1 W. S. Hummers Jr and R. E. Offeman, J. Am. Chem. Soc., 1958, 80, 1339.

2 D. C. Marcano, D. V. Kosynkin, J. M. Berlin, A. Sinitskii, Z. Sun, A. Slesarev, L. B. Alemany, W. Lu and J. M. Tour, ACS Nano, 2010, 4, 4806-4814.

3 G. Eda, Y.-Y. Lin, C. Mattevi, H. Yamaguchi, H.-A. Chen, I.-S. Chen, C.-W. Chen and M. Chhowalla, Adv. Mater., 2010, 22, 505-509.

4 Y. A. Niu, J. P. Zhao, X. Zhang, X. J. Wang, J. Wu and Y. Li, Appl. Phys. Lett., 2012, 101, 181903.
5 T. V. Cuong, V. H. Pham, Q. T. Tran, S. H. Hahn, J. S. Chung, E. W. Shin and E. J. Kim, Mater. Lett., 2010, 64, 399-401.

6 C. T. Chien, S. S. Li, W. J. Lai, Y. C. Yeh, H. A. Chen, I. S. Chen, L. C. Chen, K. H. Chen, T. Nemoto, S. Isoda, M. Chen, T. Fujita, G. Eda, H. Yamaguchi, M. Chhowalla and C. W. Chen, Angew. Chem., Int. Ed., 2012, 51, 6662-6666.

7 S. Vempati, S. Ozcan and T. Uyar, Appl. Phys. Lett., 2015, 106, 051106.

8 S. Vempati, A. Celebioglu and T. Uyar, J. Mater. Chem. C, $2014,2,8585$.

9 J. Kim, L. J. Cote, F. Kim and J. Huang, J. Am. Chem. Soc., 2010, 132, 260-267.

10 Y. Wang, D. Kurunthu, G. W. Scott and C. J. Bardeen, J. Phys. Chem. C, 2010, 114, 4153-4159.

11 H. Dong, W. Gao, F. Yan, H. Ji and H. Ju, Anal. Chem., 2010, 82, 5511-5517.

12 V. G. Plotnikov, V. A. Smirnov, M. V. Alfimov and Y. M. Shulga, High Energy Chem., 2011, 45, 411-415.

13 S. You, J. Yu, B. Sundqvist, L. A. Belyaeva, N. V. Avramenko, M. V. Korobov and A. V. Talyzin, J. Phys. Chem. C, 2013, 117, 1963-1968.

14 M. Mauro, M. Maggio, A. Antonelli, M. R. Acocella and G. Guerra, Chem. Mater., 2015, 27, 1590-1596.

15 C. Liu, Z. Wang, H. Jia and Z. Li, Chem. Commun., 2011, 47, 4661-4663.

16 C. Galande, A. D. Mohite, A. V. Naumov, W. Gao, L. Ci, A. Ajayan, H. Gao, A. Srivastava, R. B. Weisman and P. M. Ajayan, Sci. Rep., 2011, 1, 85.

17 S. Vempati and T. Uyar, Phys. Chem. Chem. Phys., 2014, 16, 21183.

18 A. Lerf, H. He, T. Riedl, M. Forster and J. Klinowski, Solid State Ionics, 1997, 101, 857-862.

19 G. Eda, C. Mattevi, H. Yamaguchi, H. K. Kim and M. Chhowalla, J. Phys. Chem. C, 2009, 113, 15768-15771.

20 X. L. Hou, J. Li, S. C. Drew, B. Tang, L. Sun and X. G. Wang, J. Phys. Chem. C, 2013, 117, 6788-6793.

21 R. Flyunt, W. Knolle, A. Kahnt, C. E. Halbig, A. Lotnyk, T. Häupl, A. Prager, S. Eigler and B. Abel, Nanoscale, 2016, 8, 7572-7579.

22 J. R. Rani, J. Lim, J. Oh, J.-W. Kim, H. S. Shin, J. H. Kim, S. Lee and S. C. Jun, J. Phys. Chem. C, 2012, 116, 19010-19017.

23 H.-K. Jeong, Y. P. Lee, R. J. W. E. Lahaye, M.-H. Park, K. H. An, I. J. Kim, C.-W. Yang, C. Y. Park, R. S. Ruoff and Y. H. Lee, J. Am. Chem. Soc., 2008, 130, 1362-1366.

24 K. Krishnamoorthy, M. Veerapandian, K. Yun and S.-J. Kim, Carbon, 2013, 53, 38-49.

25 S. Claramunt, A. Varea, D. López-Díaz, M. M. Velázquez, A. Cornet and A. Cirera, J. Phys. Chem. C, 2015, 119, 10123-10129.

26 J. Robertson, Mater. Sci. Eng., R, 2002, 37, 129-281.

27 S. Saxena, T. A. Tyson, S. Shukla, E. Negusse, H. Chen and J. Bai, Appl. Phys. Lett., 2011, 99, 013104. 
28 D. Li, M. B. Müller, S. Gilje, R. B. Kaner and G. G. Wallace, Nat. Nanotechnol., 2008, 3, 101-105.

29 A. J. Pollard, B. Brennan, H. Stec, B. J. Tyler, M. P. Seah, I. S. Gilmore and D. Roy, Appl. Phys. Lett., 2014, 105, 253107.

30 S. Pisana, M. Lazzeri, C. Casiraghi, K. Novoselov, A. K. Geim, A. C. Ferrari and F. Mauri, Nat. Mater., 2007, 6, 198.

31 A. C. Ferrari and J. Robertson, Phys. Rev. B: Condens. Matter Mater. Phys., 2000, 61, 14095-14107.
32 In a zero order approximation, we assume that the widths of the bands do not change as we reduce the sample. Hence the yield is qualitative only.

33 A. C. Ferrari and J. Robertson, Phys. Rev. B: Condens. Matter Mater. Phys., 2001, 64, 075414.

34 F. P. Schwarz and S. P. Wasik, Anal. Chem., 1976, 48, 524-528.

35 D. R. Dreyer, S. Park, C. W. Bielawski and R. S. Ruoff, Chem. Soc. Rev., 2009, 39, 228-240. 